Underestimating acid reflux

\section{Are we underestimating acid reflux?}

\section{S J Spechler}

\section{Have conventional $\mathrm{pH}$ monitoring techniques substantially underestimated the duration of acid exposure for the most distal segment of the oesophagus?}

F or a simple organ like the oesophagus, which functions primarily as a conduit, it is hard to imagine a more unpleasant neighbour than the stomach, a reservoir of concentrated acid constrained by a leaky valve. Studies using $\mathrm{pH}$ monitoring techniques developed in the 1970s have shown that the distal oesophagus of normal ambulatory individuals can be exposed to refluxed gastric acid (with $\mathrm{pH}<4$ ) for up to $5 \%$ of a 24 hour monitoring period. ${ }^{1} \quad$ Conventionally, oesophageal $\mathrm{pH}$ monitoring has been performed with the $\mathrm{pH}$ sensor positioned at a level $5 \mathrm{~cm}$ above the lower oesophageal sphincter (LOS). In a study reported in this issue of Gut, Fletcher and colleagues ${ }^{2}$ in Glasgow have found that conventional $\mathrm{pH}$ monitoring techniques have substantially underestimated the duration of acid exposure for the most distal segment of the oesophagus [see page 168].

To study acid exposure in the most distal oesophagus, the investigators devised a novel endoscopic technique for positioning a modified $\mathrm{pH}$ catheter, with two $\mathrm{pH}$ electrodes located $5 \mathrm{~cm}$ apart. A short prolene loop was attached to the catheter $5 \mathrm{~mm}$ below the distal $\mathrm{pH}$ electrode and the catheter was passed through the nose into the oesophagus. An endoscopic examination was performed, the junction of squamous and columnar epithelia (the $\mathrm{Z}$ line) in the distal oesophagus was identified, and an endoscopic clip was used to fix the prolene loop to the $\mathrm{Z}$ line. In this way, the distal pH electrode was anchored in the distal oesophagus at a level $5 \mathrm{~mm}$ above the $\mathrm{Z}$ line whereas the proximal electrode was located at approximately the conventional $\mathrm{pH}$ monitoring level.

The study group comprised 11 patients who previously had normal oesophagoscopic examinations and normal conventional 24 hour oesophageal $\mathrm{pH}$ monitoring tests. Using a combination of manometric and endoscopic measurements, the investigators determined that the distal oesophageal $\mathrm{pH}$ electrode (anchored $5 \mathrm{~mm}$ above the $\mathrm{Z}$ line) was located a mean of $6 \mathrm{~mm}$ below the upper border of the LOS. Thus the distal electrode was recording the $\mathrm{pH}$ of fluid bathing oesophageal squamous epithelium within the LOS itself.

The distal electrode recorded an oesophageal $\mathrm{pH}$ value $<4$ for a median of $11.7 \%$ of the 24 hour monitoring period whereas $5 \mathrm{~cm}$ proximally, oesophageal $\mathrm{pH}$ remained $<4$ for only $1.8 \%$ of the same period $(\mathrm{p}<0.001)$. Longer distal oesophageal acid exposures were observed both when the subjects were upright $(12.7 \% \vee 2.3 \%$; $\mathrm{p}<0.001)$ and supine $(10.5 \% v 1.3 \% ; \mathrm{p}<0.001)$, and the total number of individual reflux episodes was also greater distally ( $168 v 33$; $\mathrm{p}<0.001)$. The difference between distal and proximal acid exposures was especially prominent during the three hour period after dinner $(21.8 \% v 2.8 \%)$. Thus the distal oesophageal $\mathrm{pH}$ electrode, tethered $5 \mathrm{~mm}$ above the squamocolumnar junction and within the LOS, recorded considerably greater acid exposure than the electrode located just $5 \mathrm{~cm}$ proximally in the body of the oesophagus. In patients who have no signs of gastro-oesophageal reflux disease (GORD), this study shows that the most distal oesophagus is exposed to concentrated acid for more than $10 \%$ of the day. The authors suggest the term "short segment reflux" to describe this phenomenon.

This study by Fletcher and colleagues ${ }^{2}$ is another in a series of fascinating investigations from Glasgow elucidating how hostile is the environment at the inspiration for these studies was an interesting observation about postprandial acid reflux made in subjects who had sensors recording $\mathrm{pH}$ simultaneously in the oesophagus and in the body of the stomach. ${ }^{3}$ After a meal, the investigators noted that acid which refluxed into the oesophagus often had a $\mathrm{pH}$ value substantially lower than that recorded by the electrode in the gastric body. This observation suggested that the acid reflux was coming from a pocket of acid in the proximal stomach that had somehow escaped the buffering effects of ingested food. To confirm the presence of this postprandial acid gastro-oesophageal junction (GOJ). The pocket, pH was measured systematically at various levels throughout the stomach and oesophagus of healthy volunteers. Whereas $\mathrm{pH}$ in the body of the stomach reached a median level of 4.7 after a meal, an acid pocket with a median $\mathrm{pH}$ of 1.6 was found at the GOJ. This postprandial acid pocket had a mean length of $2 \mathrm{~cm}$, beginning in the most proximal stomach and extending more than $1 \mathrm{~cm}$ above the $\mathrm{Z}$ line into the distal oesophagus.

The mechanisms underlying the intense acid exposure of the most distal oesophagus are not known. During conventional oesophageal $\mathrm{pH}$ monitoring in which the $\mathrm{pH}$ electrode is positioned $5 \mathrm{~cm}$ above the LOS, most episodes of acid reflux are attributed to transient LOS relaxations (TLOSRs). ${ }^{4}$ Although Fletcher and colleagues ${ }^{2}$ did not monitor TLOSRs during their study, two important observations suggest that TLOSRs may not be the predominant mechanism for short segment reflux. Firstly, TLOSRs occur at an average rate of only 2-6 episodes per hour ${ }^{5}$ whereas the investigators observed approximately 7 episodes of short segment reflux per hour. Secondly, the frequency of TLOSRs decreases substantially at night (supine posture) whereas the investigators found no substantial decrease in the supine frequency of short segment reflux.

One potential mechanism for short segment reflux relates to the mechanical effect of gastric distension on the LOS. It has been proposed that gastric distension induced by eating and other factors can cause the distal portion of the LOS to be prized apart by the expanding gastric fundus. ${ }^{6}$ In this situation, the proximal portion of the sphincter remains closed, preventing reflux into the proximal oesophagus, but the distal portion is pulled open, thus exposing the most distal oesophagus to the gastric contents. The observation that short segment reflux is especially common after meals is consistent with this mechanism. Nevertheless, further studies are necessary to establish the physiology of short segment reflux.

Potential consequences of short segment reflux include not only acid peptic injury but also exposure of the distal oesophagus to high concentrations of nitric oxide (NO) generated from dietary nitrate $\left(\mathrm{NO}_{3}{ }^{-}\right)$in green leafy vegetables. Most ingested nitrate is absorbed by the small intestine and excreted unchanged in the urine, but approximately $25 \%$ is concentrated by the salivary glands and secreted into the mouth where bacteria on the tongue reduce the recycled nitrate to nitrite $\left(\mathrm{NO}_{2}{ }^{-}\right) .^{78}$ When swallowed, nitrite encounters acidic gastric juice and the nitrite is converted rapidly 
to NO. ${ }^{9}{ }^{10}$ In earlier studies, the Glasgow group demonstrated that high levels of NO are generated at the GOJ, the region where swallowed nitrite first meets gastric acid. ${ }^{11} \mathrm{NO}$ can participate in nitrosation reactions that result in the deamination of the purine and pyrimidine bases of DNA, the formation of carcinogenic $\mathrm{N}$-nitroso compounds from amines and amides, and the inactivation of DNA repair enzymes. ${ }^{12-14} \mathrm{NO}$ also can participate in redox reactions which generate reactive oxygen species that can further damage DNA. ${ }^{15}$ Thus NO can be genotoxic and, potentially, carcinogenic.

This study by Fletcher and colleagues ${ }^{2}$ suggests that short segment reflux causes relentless exposure of the most distal oesophagus to acid, NO, and other noxious agents in gastric juice. Intestinal metaplasia at the end of the oesophagus (short segment Barrett's oesophagus) is a common condition, even in patients who have no signs or symptoms of GORD. ${ }^{16}$ Intestinal metaplasia, a sequela of chronic inflammation, can predispose to cancer. ${ }^{16}{ }^{17}$ It seems likely that short segment reflux can cause chronic distal oesophagitis that contributes to the pathogenesis of short segment Barrett's oesophagus and, ultimately, oesophageal adenocarcinoma, a tumour whose frequency has been rising at an impressive rate for decades. ${ }^{18}$ Nevertheless, oesophageal adenocarcinoma remains an uncommon tumour whereas short segment reflux appears to be a common, perhaps even normal, phenomenon. Considering the great frequency of short segment reflux, the fact that short segment Barrett's oesophagus occurs so commonly perhaps is less remarkable than the fact that serious peptic and neoplastic complications of GORD occur so infrequently. Apparently, the squamous epithelium of the most distal oesophagus has ample intrinsic defence mechanisms and considerable capacity to resist injury from the noxious brew spewed by its unpleasant neighbour to the south. To paraphrase Robert Frost, good defences make good neighbours.

Gut 2004;53:162-163

Correspondence to: Dr S J Spechler, Division of Gastroenterology (111 B 1), Dallas VA Medical Center, 4500 South Lancaster Rd, Dallas, Texas 75216, USA; SJSpechler@AOL.com

\section{REFERENCES}

1 Richter JE, Bradley LA, DeMeester TR, et al. Normal 24-hr ambulatory esophageal $\mathrm{pH}$ values. Influence of study center, $\mathrm{pH}$ electrode, age, and gender. Dig Dis Sci 1992;37:849-56.

2 Fletcher J, Wirz A, Henry E, et al. Studies of acid exposure immediately above the gastrooesophageal squamocolumnar junction: evidence of short segment reflux. Gut 2003;53:168-73.

3 Fletcher J, Wirz A, Young J, et al. Unbuffered highly acidic gastric juice exists at the gastroesophageal junction after a meal. Gastroenterology 2001;121:775-83.

4 Schoeman MN, Tippett MD, Akkermans LMA, et al. Mechanisms of gastroesophageal reflux in ambulant healthy human subjects. Gastroenterology 1995;108:83-91.

5 Mittal RK, Holloway RH, Penagini R, et al. Transient lower esophageal sphincter relaxation. Gastroenterology 1995;109:601-10.
6 Oberg S, Peters JH, DeMeester TR, et al Inflammation and specialized intestinal metaplasia of cardiac mucosa is a manifestation of gastroesophageal reflux disease. Ann Surg 1997;226:522-32.

7 Bartholomew B, Hill MJ. The pharmacology of dietary nitrate and the origin of urinary nitrate. Food Chem Toxicol 1984;22:789-95.

8 Duncan C, Dougall H, Johnston P, et al. Chemical generation of nitric oxide in the mouth from the enterosalivary circulation of dietary nitrate. Nat Med 1995; 1:546-51.

9 Moriya A, Grant J, Mowat C, et al. In vitro studies indicate that acid catalysed generation of $\mathrm{N}$ nitrosocompounds from dietary nitrate will be maximal at the gastro-oesophageal junction and cardia. Scand J Gastroenterol 2002;37:253-61.

10 lijima K, Fyfe V, McColl KEL. Studies of nitric oxide generation from salivary nitrite in human gastric juice. Scand J Gastroenterol 2003;38:246-52.

11 lijima K, Henry E, Moriya A, et al. Dietary nitrate generates potentially mutagenic concentrations of nitric oxide at the gastroesophageal junction. Gastroenterology 2002;122:1248-57.

12 Liu RH, Hotchkiss JH. Potential genotoxicity of chronically elevated nitric oxide: a review. Mutat Res 1995; 339:73-89.

13 Felley-Bosco E. Role of nitric oxide in genotoxicity: implications for carcinogenesis. Cancer Metastasis Rev 1998:17:25-37.

14 Jaiswal M, LaRusso NF, Burgart $\amalg$, et al. Inflammatory cytokines induce DNA damage and inhibit DNA repair in cholangiocarcinoma cells by a nitric oxide-dependent mechanism. Cancer Res 2000;60:184-90.

15 Stamler JS, Singel DJ, Loscalzo J. Biochemistry of nitric oxide and its redox-activated forms. Science 1992; 258: 1898-902

16 Spechler SJ. The role of gastric carditis in metaplasia and neoplasia at the gastroesophageal junction. Gastroenterology 1999:117:218-28.

17 Spechler SJ. Laser photoablation of Barrett's epithelium: burning issues about burning tissues. Gastroenterology 1993;104:1855-8.

18 Brown LM, Devesa SS. Epidemiologic trends in esophageal and gastric cancer in the United States. Surg Oncol Clin N Am 2002;11:235-56.

\section{A snapshot of colonoscopy practice in England: stimulus for improvement}

\section{K R Palmer, A I Morris}

\section{Colonoscopy practice in the UK today: are we adequately prepared for national colorectal cancer screening tomorrow?}

som the West, $5 \%$ of individuals will at some time in their life develop colon cancer, and after the age of 54 years the risk lies between 1 in 19 to 1 in $22 .{ }^{12}$ There is increasing pressure to develop colon screening programmes to identify early tumours and adenomatous polyps whose endoscopic removal has been shown to reduce cancer development. ${ }^{34}$ Colon cancer screening is likely to be funded in England and in Scotland, and whether this is based on faecal occult blood testing, ${ }^{5}{ }^{6}$ flexible sigmoidoscopy, or colonoscopy alone, ${ }^{89}$ and whether or not programmes will be targeted towards high risk groups or to all comers over a specific age, it is obvious that referrals for colonoscopy will increase. At the same time, there is increasing awareness among the general population and in primary care that rectal bleeding and altered bowel habit need investigation, ideally by colonoscopy. Most endoscopy units in the UK have difficulty coping with their current workload, and strains on the colonoscopy waiting list will inevitably increase when screening programmes are instigated.

Series published from the USA have reported success rates in excess of $97 \%$ for achieving total colonoscopy, coupled with low complication rates, and detection of significant pathology in asymptomatic populations. ${ }^{810}$ Specialist centres in the UK report comparable data and have shown that it is possible to undertake safe diagnostic and therapeutic colonoscopy using minimal sedation, little patient discomfort, and low complication rates. The Joint Advisory Group on Gastrointestinal Endoscopy (JAG) state that approved endoscopy units should achieve completion rates greater than $90 \%$, without 
resort to excessive sedation. The current curriculum for higher medical training in gastroenterology suggests that trainees should demonstrate this level of expertise at the time of completion of specialist training. ${ }^{11}$

In the light of these observations, the snapshot of colonoscopy practice in 68 English units, reported in this use of Gut, $^{12}$ is disturbing [see page 277]. Caecal intubation rates were $77 \%$, falling to $57 \%$ when the strict criteria of appendiceal orifice identification or intubation of the ileocaecal valve were used. The perforation rate was 1:769, and it is likely that colonoscopy contributed to the death of six patients. There are several possible mitigating factors. It is not clear for example whether it was the intention in every case to achieve pancolonoscopy; it is possible to speculate that in some cases the intention was to identify a relatively distal abnormality and it was then deemed unnecessary to visualise the whole colon. Criteria for completion may not have been defined prior to the audit and, while it is now standard practice to identify the appropriate landmarks to confirm completion rather than to rely on transillumination or indentation, this was probably not universal at the time of data collection. Furthermore, the case mix of patients in this series is likely to have been different to that reported from some other sources; for example, colonoscopy done in relatively young asymptomatic individuals as part of colon cancer screening programmes is generally more straightforward than that in patients who present with colonic symptoms-as was the case in the audit. These are however relatively weak arguments; pancolonoscopy should be the aim in all patients, the credibility of transillumination and indentation are flawed, and good colonoscopists succeed in the great majority of patients. It is noteworthy that the common reasons cited for failure were excessive looping and patient discomfort, aspects of practice minimised by good technique. Lastly, were the units who took part in the study particularly incompetent, chosen because they were known for their modest expertise? Obviously this was not the case; institutions who are motivated enough to participate in clinical research tend to be the better ones and it is therefore very likely that the picture painted in the paper is a reasonable, perhaps even an optimistic, representation of colonoscopy practice in the UK.

What then should British endoscopists do to rectify the unsatisfactory situation, particularly with the prospect of colonoscopy screening around the corner? As it is, we are apparently in danger of causing net harm to the general population by undertaking colonoscopy programmes with a possible $0.1 \%$ perforation rate and $0.05 \%$ mortality. In response, medical and surgical endoscopists have, even since the audit data were collected, improved aspects of expertise and training in endoscopy and many of the issues highlighted in the audit are being actively addressed.

The improvements started with the formation of JAG in 1994 by the Conference of Royal Colleges, and its publication of a first document in 1999 entitled Recommendations for training in gastrointestinal endoscopy. Within three years a second document, entitled Guidelines for the training, appraisal and assessment of trainees in GI endoscopy, was published. This emphasised achievement of targets and competence, rather than simply completing set numbers of procedures. Laid down in this document was the requirement that trainee endoscopists, from whatever discipline (medicine, surgery, non-medical), were to be trained to the same standards and that they and their trainers were required to attend proscribed JAG courses.

JAG specified that all trainees should attend a basic skills (foundation) course, followed by other specific courses related to the particular endoscopic skill they were learning. The Raven Department of Education at the Royal College of Surgeons of England was commissioned to develop the courses for JAG. Thus far, in addition to the foundation course, which provides "hands on" gastroscopy training, a basic skills in colonoscopy, and flexible sigmoidoscopy courses (also "hands on" courses) have been developed. Therapeutic endoscopy, ERCP, and EUS courses (partly funded by the British Society of Gastroenterology) are soon to be commissioned. Trainers were also to attend specific training the trainers courses for endoscopy when these were available.

A total of 250 trainees have so far attended the colonoscopy courses that are currently delivered at three full time and seven part time centres. There is no doubt that these "hands on" learning experiences are effective as the colonoscopy completion rate of those individuals who have attended a course is well in excess of $90 \%$. These courses have to date been supported to the sum of $£ 2.4$ million for the period 2001-2003 from the National Cancer Project.

This year marks four major initiatives concerned with training in endoscopy.

The first major undertaking will be publishing of the third JAG document with new sections devoted to the assessment of both trainees and training units.
The second major development has been a government allocation of $£ 8.2$ million, via the National Cancer Plan, to support endoscopy training in England over the years 2003-2006. This has funded three national training centres (based at the Royal Liverpool Hospital, St George's Hospital, and St Mark's Hospitals) and seven regional endoscopy training centres, and is administered by the NHS Modernisation Agency Endoscopy Project. Service improvements which aspire to improve efficiency, quality, and output are developed in parallel with endoscopy training.

The third development has been the revision of the endoscopy section of the specialist medical curriculum for higher medical training in gastroenterology, produced by the statutory Specialist Advisory Committees (SACs) on behalf of the Joint Committee on Higher Medical Training. This is now a competency based assessment devised by the JAG.

Fourthly, JAG has developed a system for accrediting new endoscopy units and re-accrediting established units. A programme of inspections, held in conjunction with the SAC in gastroenterology, is planned to start in April 2004. These visits will concentrate on the training environment and will include scrutiny of endoscopy standards and compliance with current national guidelines. In order to limit the number of visitations to units, it is intended to involve the endoscopy lead for each strategic health authority in the visits so that aspects of service delivery can be assessed.

It will take some time before all endoscopists are trained to appropriate standards and all units are optimally equipped and functioning. We anticipate that colonoscopy practice in the UK will improve but it is crucial for our credibility that this is confirmed by future surveys of practice. The Department of Health seems remarkably unenthusiastic to support large scale audits of gastrointestinal practice; there has been no success in obtaining government funding for our audit of ERCP and attempts to support further audits of upper gastrointestinal bleeding, acute pancreatitis, and management of inflammatory bowel disease seem futile at the time of writing. The results of the colonoscopy audit will undoubtedly stimulate adverse comment about British endoscopy performance, but the audit has already caused us-and the Department of Health - to address important issues of training, equipment, and facilities. Surely we must strive for more large scale properly supported audits in order to improve and justify our practice, not only in relation to endoscopy but for the full range of our activities within gastroenterology. 
Gut 2004;53:163-165.

doi: $10.1136 /$ gut.2003.022053

\section{Authors' affiliations \\ K Palmer, British Society of Gastroenterology, London, UK \\ A I Morris, Joint Advisory Group on Gastrointestinal Endoscopy, London, UK \\ Correspondence to: Dr K R Palmer, Western General Hospital, Crewe Rd, Edinburgh EH4 2XU, UK; kpalmer@golf5063.freeserve.co.uk \\ REFERENCES \\ 1 Cancer Surveillance Team. Scottish Cancer Intelligence Group, Information and Statistics Division. Scotland: NHS. \\ 2 Ransahoff DF. Colorectal cancer. In: Everhart JE eds. Digestive diseases of the United States:}

epidemiology and impact. Washington, DC: US Department of Health and Human Sciences, Public Health Service National Institute of Health, National Institute of Diabetes and Digestive and Kidney Diseases, US Government Printing Office, 1994:205-224, NIH publications No 94-1447.

3 Selby JV, Friedman GD, Quesenberry GP jr, et al. A case controlled study of screening sigmoidoscopy and mortality from colorectal cancer. N Engl J Med 1992;326:653.

4 Winawer SJ, Zauber AG, Ho MN, et al. Prevention of colorectal cancer by colonoscopic polypectomy. The National study workgroup. N Engl J Med 1993;329:1977-81.

5 Mandel JS, Bond JH, Church TR, et al. Reducing mortality from colorectal cancer by screening for fecal occult blood. N Engl J Med 1993:328:1365-71.

6 Hardcastle JD, Chamberlain JO, Robinson MHF, et al. Randomised controlled trial of faecal occult blood screening for colorectal cancer. Lancet 1996;348:1472-7.
7 Imperale TF, Wagner DR, Ching Y, et al. Risk of advanced proximal neoplasm in asymptomatic adults according to the distal colorectal findings. N Engl J Med 200;343:169-74.

8 Lieberman DA, Weiss DG, Bond JH, et al. Use of colonoscopy to screen asymptomatic adults for colorectal carcinoma. N Engl J Med 2000;343:162-8.

$9 \operatorname{Rex} \mathrm{D}$, Johnson DA, Lieberman DA, et al. Colorectal cancer prevention 2000; screening recommendations of the American College of Gastroenterology. Am J Gastroenterol 2000;95:868-77.

10 Nelson DB, McQuaid KR, Bond JR, et al. Procedural success and complications of large scale screening colonoscopy. Gastrointest Endosc 2002;55:307-14

11 JCHMT. Higher Medical Training Committee for Gastroenterology. London: JCHMT, 2003.

12 Bowles CJA, Leicester R, Romaya C, et al. A prospective study of colonoscopy practice in the UK today: are we adequately prepared for national colorectal cancer screening tomorrow? Gut 2004;53:277-83.

\title{
Surveillance colonoscopy in ulcerative colitis: magnifying chromoendoscopy in the spotlight
}

\author{
R Kiesslich, M F Neurath
}

Chromoendoscopy in ulcerative colitis may offer the possibility of detecting dysplastic or neoplastic changes at a curable stage, and thus expand the indication for chromoendoscopy from screening to surveillance colonoscopy

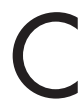

olonoscopy is the gold standard for colorectal cancer screening. ${ }^{1-3}$ of adenomas disrupt the adenomacarcinoma sequence and thus prevent the development of colorectal cancer. However, endoscopists still fear that they may have overlooked relevant lesions despite the availability of modern videoendoscopes. This problem is underlined by a relatively high rate of adenomas missed by conventional endoscopy (up to $27 \%$ ), as determined by back to back or repeat colonoscopy studies. $^{23}$ Furthermore, retrospective analyses have suggested that colonoscopy may even fail to detect colorectal cancer (CRC), ${ }^{45}$ although large multicentre studies indicated a high negative predictive value for a normal complete colonoscopy with regard to CRC (>99\%). ${ }^{6}$ Therefore, the question arises of whether adenomas or CRC detected after previous colonoscopies have grown fast or were simply overlooked during initial endoscopic analysis?

Exophytic adenomas can be diagnosed easily and most of the Western endoscopists have previously focused on these polypoid lesions. In contrast, several years ago Japanese researchers described flat lesions in the colonic mucosa and classified these so-called "flat adenomas" as premalignant lesions. $^{78}$ These lesions now can be subdivided according to their growing pattern: small flat adenoma, lateral spreading adenoma, and depressed lesions with high malignant potential. It is well accepted that flat lesions show only small changes in the mucosal architecture, such as small depressions and discrete changes in colour. Detection of such subtle mucosal changes during conventional colonoscopy remained a challenge, even for experienced endoscopists. Recently, using dye spraying techniques, chromoendoscopy has revolutionised the detection of flat lesions in the colonic mucosa and, when used in a targeted fashion, allows the unmasking of the type of lesion and its borderlines. Furthermore, the use of magnifying endoscopes during chromoendoscopy allows a detailed surface analysis of suspected lesions and prediction of the dignity of the lesions using the so-called pit pattern classification (see fig 1). These features of magnifying chromoendoscopy may also offer the possibility of achieving appropriate diagnostic and therapeutic decisions during ongoing colonoscopies (for example, biopsy, polypectomy, and mucosal resection versus surgery).

Depressed lesions can lead to early malignant invasion, even when small in size. For a long time these lesions were frequently not recognised by Western endoscopists. However, Fujii and colleagues $^{9}$ impressively demonstrated that detection of depressed early colorectal malignancies was possible with the help of chromoendoscopy and magnification endoscopy in the UK. This first observation was confirmed by Rembacken and colleagues. ${ }^{10}$ After training in chromoand magnifying endoscopy, the investigators showed that in 1000 patients, 117 flat and two depressed lesions among 321 adenomas could be diagnosed. Similar results were seen by Saitoh and colleagues ${ }^{11}$ where American and Japanese endoscopists performed team colonoscopies in 211 American patients and stained parts or the whole colon with indigo carmine. In $23 \%$ of cases, flat lesions or flat lesions with depressions were diagnosed. Most of these lesions $(82 \%)$ were adenomatous. In conclusion, these data show clearly that intravital staining techniques allow significantly improved detection and analysis of flat lesions and should be part of the endoscopist's armamentarium. ${ }^{12}$ The endoscopist should be aware of diminutive mucosal alterations and stain in a targeted fashion, thereby reducing the additional time required to perform magnifying chromoendoscopy and increasing the diagnostic yield for screening colonoscopy in CRC. 
A

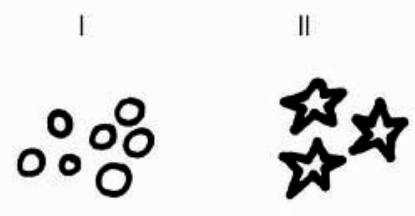

Non-neoplastic pattern

B

Example type I

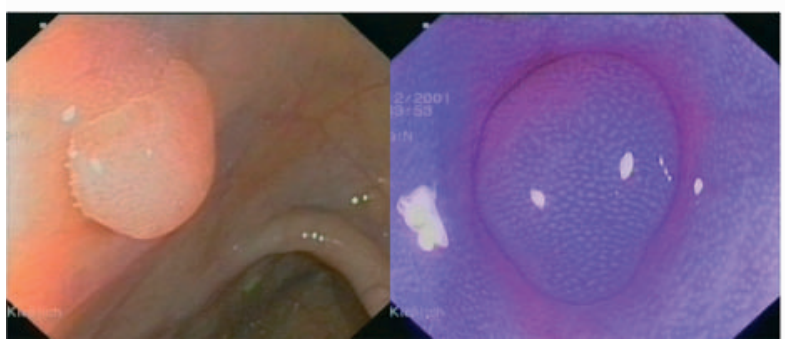

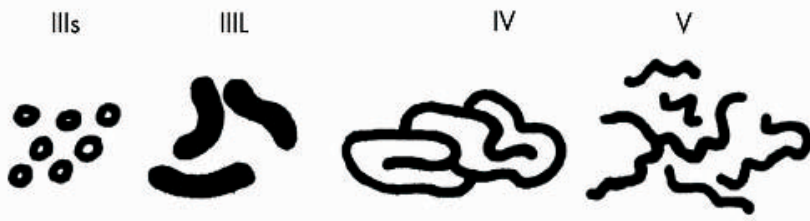

Neoplastic pattern

Example type IV

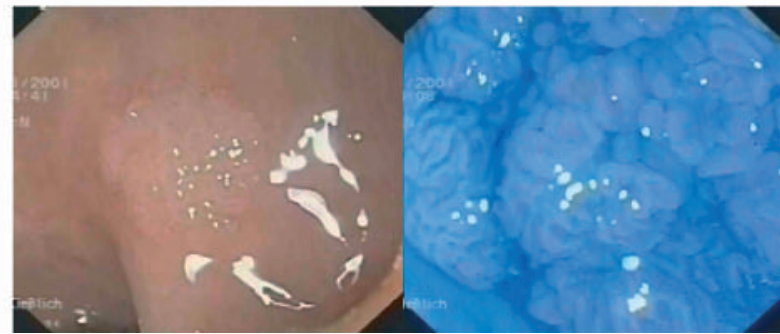

Figure 1 Pit pattern classification according to Kudo and colleagues. ${ }^{16}$. The typical crypt architecture of types I-V are indicated (A). (B) Examples of type I (left) and type IV (right) lesions before and after chromoendoscopy.

In this issue of Gut, Rutter and colleagues $^{13}$ describe the higher sensitivity of pancolonic chromoendoscopy with indigo carmine dye spraying for the detection of intraepithelial neoplasias in patients with longstanding ulcerative colitis (UC) [see page 256]. The collective of patients with UC is ideal for intravital staining. Patients with chronic active UC have an increased risk of colitis associated colon cancer, depending on the duration, extent, and activity of the disease, and the presence of primary sclerosing cholangitis, and this has led to considerable efforts to detect early lesions in UC. ${ }^{14-19}$ Furthermore, in contrast with sporadic CRC, lesions in UC patients do not follow the classical adenoma-carcinoma sequence but colitis associated dysplastic changes are first limited to the mucosa and submucosa and the growing pattern of dysplastic changes is often multifocal and flat. Thus chromoendoscopy in UC patients should be used in an extensive (panchromoendoscopy) rather than in a targeted fashion. In conjunction with magnifying endoscopes, chromoendoscopy in UC may offer the possibility of detecting dysplastic or neoplastic changes at a curable stage, and thus expand the indication for chromoendoscopy from screening to surveillance colonoscopy.

Rutter and colleagues ${ }^{13}$ investigated 100 patients with back to back colonoscopy, starting with random and targeted biopsies followed by panchromoendoscopy with targeted biopsies only. The design of this study is interesting although the potential value of chromoendoscopy after intensive random biopsies may even have been underestimated due to multiple bleedings that might have interfered with the chromoendoscopy procedure. But despite this potential limitation, the diagnostic yield of dysplastic changes was increased in this study by the use of chromoendoscopy from two to seven patients (3.5-fold increase) and from two to nine dysplastic lesions (4.5-fold increase). After panchromoendoscopy, a plethora of lesions were unmasked in UC patients. The majority of the lesions consisted of non-neoplastic tissue but this is a common problem using chromoendoscopy in a non-targeted fashion ${ }^{15}$ and endoscopists need to select and focus towards lesions with suspicious surface staining patterns.

New powerful endoscopes (high resolution and magnifying endoscopes) offer resolutions which allow new surface details to be seen on the colonic mucosa, such as the opening of the crypts. Kudo and colleagues ${ }^{16}$ were the first to note that some of the regular staining patterns were often seen in hyperplastic polyps or normal mucosa whereas unstructured surface architecture was associated with malignancy. This experience has led to categorisation of the different staining patterns in the colon. The so-called pit pattern classification $^{16}$ differentiated five types and several subtypes. Types I and II are staining patterns that predict non-neoplastic lesions whereas types III to V predict neoplastic lesions (fig 1 ). With the help of this classification, the endoscopist can predict histology with good accuracy.

The study by Rutter et al demonstrates improved detection of colonic lesions in UC patients using chromoendoscopy with indigo carmine. This study strongly supports and extends recent studies in UC patients. In a randomised controlled trial using magnifying chromoendoscopy in patients with longstanding UC, we showed that the diagnostic yield for intraepithelial neoplasias was significantly increased by using methylene blue staining. ${ }^{18}$ More targeted biopsies were possible, and significantly more intraepithelial neoplasias were detected in the chromoendoscopy group. Using the modified pit pattern classification (pit patterns I-II: endoscopic prediction-non-neoplastic; pit patterns IIIV: endoscopic prediction-neoplastic), both the sensitivity and specificity for differentiation between non-neoplastic and neoplastic lesions were $93 \%{ }^{18}$ Furthermore, Hurlstone ${ }^{19}$ recently analysed 162 patients with longstanding UC in a prospective study using magnifying chromoendoscopy with indigocarmine. After detection of subtle mucosal changes (such as fold convergence, air induced deformation, interruption of innominate grooves, or focal discrete colour change), targeted intravital staining with indigo carmine was used. Chromoendoscopy with targeted biopsy significantly increased the diagnostic yield for intraepithelial neoplasia and the number of flat neoplastic changes as opposed to conventional colonoscopy. Intraepithelial neoplasia in flat mucosal 
Table 1 Seven guidelines (SURFACE) for chromoendoscopy in ulcerative colitis

(1) Strict patient selection

Patients with histologically proven ulcerative colitis and at least eight years' duration in clinical remission. Avoid patients with active disease.

(2) Unmask the mucosal surface.

Excellent bowel preparation is needed. Remove mucus and remaining fluid in the colon when necessary. (3) Reduce peristaltic waves.

When drawing back the endoscope, a spasmolytic agent should be used (if necessary).

(4) Full length staining of the colon.

Perform full length staining of the colon (panchromoendoscopy) in ulcerative colitis rather than local staining

(5) Augmented detection with dyes.

Intravital staining with $0.4 \%$ indigo carmine or $0.1 \%$ methylene blue should be used to unmask flat lesions more frequently than with conventional colonoscopy.

(6) Crypt architecture analysis.

All lesions should be analysed according to the pit pattern classification. Whereas pit pattern types I-I suggest the presence of non-malignant lesions, staining patterns III-V suggest the presence of intraepithelial neoplasias and carcinomas.

(7) Endoscopic targeted biopsies.

Perform targeted biopsies of all mucosal alterations, particularly of circumscript lesions with staining patterns indicative of intraepithelial neoplasias and carcinomas (pit patterns III-V).

change was observed in 37 lesions, of which 31 were detected using chromoendoscopy. The overall sensitivity of magnifying chromoendoscopy in predicting neoplasia was $97 \%$ with a specificity of $93 \%$.

In patients with Barrett's oesophagus, inflammation is a frequent source of irritation for both the pathologist and the endoscopist. Thus antiinflammatory therapy with proton pump inhibitors prior to chromo- and magnification endoscopy is recommended. ${ }^{17}$ We have obtained similar experience in patients with UC. ${ }^{18}$ Therefore, we believe that patients should undergo endoscopy in clinical remission as inflammatory activity in UC may mimic neoplastic changes. Based on these findings, pit pattern analysis should be limited to circumscript lesions only, whereas diffuse alterations should be attributed to local inflammation. ${ }^{18}$

One key question resulting from the above mentioned studies is whether there is still a need for random biopsies in unsuspicious mucosa after chromoendoscopy in UC patients. This is an important question as it takes a long time to perform 30-50 random biopsies during surveillance colonoscopy in UC. In our study, dysplastic tissue was seen only in conjunction with mucosal alterations (prior or after staining). Furthermore, Rutter et al found no dysplastic tissue in 2904 non-targeted biopsies. ${ }^{13} 18$ Taken together, these data suggest that targeted biopsies after intravital staining will replace random biopsies in the future, although prospective studies fully addressing this point are required. Although panchromoendoscopy requires additional time during surveillance colonoscopy (about 8-9 minutes), ${ }^{18}$ the use of targeted rather than random biopsies will save time. Therefore, the total time required for panchromoendoscopy with targeted biopsies is similar compared with colonoscopy with random biopsies but chromoendoscopy has a higher efficacy for detection of intraepithelial neoplasia, as shown by Rutter and colleagues. ${ }^{13}$

In summary, it appears that magnifying chromoendoscopy is an evolving new standard for surveillance colonoscopy in patients with longstanding UC. However, based on the currently available data, ${ }^{13}{ }^{18-19}$ we would propose several guidelines (SURFACE) for the use of this new technique in patients with UC (see table 1). Furthermore, comparative studies will be required to determine the dye that should preferentially be used for chromoendoscopy (methylene blue $v$ indigo carmine) in UC. The ability of the dye technique to differentiate neoplastic from non-neoplastic lesions and to enhance detection of dysplastic lesions in flat mucosa is a major advance in dysplasia surveillance in UC however. With the help of this new technique, the old problem of missing flat colonic lesions in patients with UC should be overcome and more patients with intraepithelial neoplasias will be diagnosed. However, magnifying chromoendoscopy cannot solve the problem of differentiating between colitis associated dysplasia and adenoma as the staining pattern of both entities is similar. However, chromoendoscopy significantly increases the diagnostic yield of intraepithelial neoplasias in UC compared with conventional colonoscopy by 3-4-fold. Differentiation of non-neoplastic from neoplastic lesions is possible by using surface staining analysis of circumscript lesions with a high overall sensitivity and specificity. It thus appears that the golden era of magnifying chromoendoscopy in UC is just about to begin.
Gut 2004;53:165-167

\section{Authors' affiliations}

R Kiesslich, M F Neurath, I Med Clinic, Johannes Gutenberg University Mainz, Germany

Correspondence to: $\operatorname{Dr}$ M F Neurath, I. Med Klinik und Poliklinik, Johannes Gutenberg Universität Mainz, Langenbeckstr 1, 55101 Mainz Germany;

neurath@1-med.klinik.uni-mainz.de

\section{REFERENCES}

1 Lambert R, Provenzale D, Ectors N, et al. Early diagnosis and prevention of sporadic colorectal cancer. Endoscopy 2001;33:1042-64.

2 Rex DK, Cutler CS, Lemmel GT, et al.

Colonoscopic miss rates of adenomas determined by back-to-back colonoscopies. Gastroenterology 1997:112:24-8.

3 Bensen S, Mott LA, Dain B, et al. The colonoscopic miss rate and true one-year recurrence of colorectal neoplastic polyps. Polyp Prevention Study Group. Am J Gastroenterol 1999;94:194-9.

4 Haseman JH, Lemmel GT, Rahmani EY, et al. Failure of colonoscopy to detect colorectal cancer: evaluation of 47 cases in 20 hospitals. Gastrointest Endosc 1997;45:451-5.

5 Gorski TF, Rosen L, Riether R, et al. Colorectal cancer after surveillance colonoscopy: falsenegative examination or fast growth? Dis Colon Rectum 1999;42:877-80

6 Ee HC, Semmens JB, Hoffman NE, et al. Complete colonoscopy rarely misses cancer. Gastrointest Endosc 2002;55:167-71

7 Kudo S, Kashida H, Tamura T, et al. Colonoscopic diagnosis and management of nonpolypoid early colorectal cancer. World J Surg 2000;24:1081-90.

8 Ajioka Y, Watanabe H, Kazama S, et al. Early colorectal cancer with special reference to the superficial nonpolypoid type from a histopathologic point of view. World J Surg 2000;24:1075-80.

9 Fujii T, Rembacken BJ, Dixon MF, et al. Flat adenomas in the United Kingdom: are treatable cancers being missed? Endoscopy 1998;30:437-43.

10 Rembacken BJ, Fuiii T, Cairns A, et al. Flat and depressed colonic neoplasms: a prospective study of 1000 colonoscopies in the UK. Lancet 2000;8:1211-14.

11 Saitoh Y, Waxmann I, West AB, et al. Prevalence and distinctive biological features of flat colorectal adenomas in a North American population. Gastroenterology 2001;120:1657-65.

12 Fennerty MB. Should chromoscopy be part of the "proficient" endoscopist's armamentarium? Gastrointest Endosc 1998:47:313-15.

13 Rutter MD, Saunders BP, Schofield G, et al. Pancolonic indigo carmine dye spraying for the detection of dysplasia in ulcerative colitis. Gut 2003:53:256-60.

14 Messmann H, Endlicher E, Freunek G, et al Fluorescence endoscopy for the detection of low and high grade dysplasia in ulcerative colitis using systemic or local 5-aminolaevulinic acid sensitisation. Gut 2003:52:1003-7.

15 Kiesslich R, von Bergh M, Hahn M, et al. Chromoendoscopy with indigo carmine improves the detection of adenomatous and non-adenomatous lesions in the colon. Endoscopy 2001;33:1001-6.

16 Kudo S, Tamura S, Nakajima T, et al. Diagnosis of colorectal tumorous lesions by magnifying endoscopy. Gastrointest Endosc 1996;44:8-14.

17 Kiesslich R, Jung M. Magnification endoscopy: does it improve mucosal surface analysis for the diagnosis of gastrointestinal neoplasias? Endoscopy 2002;34:819-22.

18 Kiesslich R, Fritsch J, Holtmann M, et al. Methylene blue aided chromoendoscopy for the detection of intraepithelial neoplasia and colon cancer in ulcerative colitis. Gastroenterology 2003;124:880-8.

19 Hurlstone DP. Further validation of highmagnification-chromoscopic-colonoscopy for the detection of intraepithelial neoplasia and colon cancer in ulcerative colitis. Gastroenterology 2003 (in press). 